\title{
Paper Reconfigurable Antenna Arrays with Phase-only Control in the Presence of Near-field Nulls
}

\author{
Giulia Buttazzoni and Roberto Vescovo \\ Department of Engineering and Architecture, University of Trieste, Trieste, Italy
}

\begin{abstract}
In this paper an effective iterative method is presented for the power synthesis of reconfigurable antenna arrays. The algorithm is suitable for arrays of arbitrary geometry, including the case where a large number of elements is involved. The reconfigurability is achieved by phase-only control, so that the excitation amplitude of each array element remains constant during the reconfiguration process. Such amplitudes may be different from one array element to the others, and they are not assigned $a$ priori, but are optimized. Furthermore, the electric field is imposed to vanish in a number of prescribed points of the near-field region, so that a strong field reduction is obtained in a neighborhood of them.
\end{abstract}

Keywords-antenna arrays, near-field nulls, phase-only control, power synthesis, reconfigurability.

\section{Introduction}

Nowadays, antenna arrays consisting of many elements are very common structures in several applications, such as radars [1], [2], satellites [3], [4] and wireless communications [5], [6]. One of their attracting features is the reconfigurability, that is, the capability of generating different radiation patterns by suitably modifying parameters, such as for example the position and/or the excitation of the radiating elements, so that many patterns can be radiated by a single antenna. The position-control, also known as mechanical steering, requires a mechanical driving system and is not very well suited in some applications such as automotive and aeronautical ones. This makes fully electronically steerable structures more interesting. Modifying both the amplitude and phase of the excitations yields many degrees of freedom, but may require the use of expensive feeding networks. Usually, the reconfigurability from one pattern to another is achieved by modifying only the phase of the excitations. This allows the use of simpler feeding networks. Many techniques have been proposed for the power pattern synthesis of reconfigurable antenna arrays with phase-only control [1], [7]-[13]. In [7], [8] a pre-assigned amplitude distribution is assumed and only the optimum values for the phase are calculated. This transforms an inherently coupled problem into a number of (simpler) independent synthesis problems (one for each pattern), but provides a non-optimal solution because the pre-assigned amplitude distribution is not the optimal one. A research of a common amplitude distribution, in conjunction with the combination of a suitable phase distribution, is more convenient [1], [9]-[13]. Besides, antenna arrays are often mounted in complex environments, such as for example ships, aircrafts and satellites, thus involving obstacles which can interfere with the far-field patterns. It is important to take into account the effects of the environment on the radiation patterns. This can be done in two ways. The first one consists in including the environment in which the antenna is operating into the synthesis procedure [14], [15]. This approach, however, requires a detailed material and geometrical description of the operating environment, as well as a significant modification of the numerical code for the pattern evaluation, necessary to evaluate the electromagnetic coupling. The second approach consists in reducing the radiated field in the zone where the obstacle is located, in order to isolate it. The obstacle isolation can be realized by minimizing the power radiated into the near-field region that includes the obstacle [16]-[19]], or by imposing an upper bound on the electric-field amplitude in the region of interest [20]-[24], or finally by imposing that the near-field vanishes in suitably chosen points [25]-[27], thus reducing the field in a neighborhood of such points. It has been demonstrated [16] that both these two approaches give satisfactory results. However, the second one (i.e., reducing the radiated field in a given zone) is much simpler. But although the near-field constraint has an increasing relevance in practical applications involving arrays, only [19]-[21], [23] propose synthesis techniques for reconfigurable arrays involving near-field constraints, and following the above second approach.

The algorithm proposed in this paper allows to synthesize a number of desired patterns for phase-only reconfigurable antenna arrays of arbitrary geometry, in such a way that the near-fields corresponding to all of the synthesized patterns vanish at a prescribed number of points close to the antenna. This results in a reduction of the near-field amplitude also in a neighborhood of these points.

\section{The Problem Formulation and the Solving Procedure}

With reference to a Cartesian system $O(x, y, z)$, let us consider an antenna array of arbitrary geometry consisting of 
$N$ arbitrary radiating elements. We want to find $S$ (complex) excitation vectors $\mathbf{i}_{s}=\left[i_{1 s}, \ldots, i_{N s}\right]^{T}$ such that the $S$ corresponding radiation patterns $F\left(\mathbf{i}_{s} ; \varphi\right)$, where $\varphi$ is the azimuth angle of the generic direction of the $x y$-plane, have a desired shape. This requirement is obtained by imposing that the $s$-th pattern belong to a suitable mask $K_{s}=$ $\left\{f_{s}(\varphi): K_{s}^{1}(\varphi) \leq\left|f_{s}(\varphi)\right| \leq K_{s}^{2}(\varphi),-\pi \leq \varphi \leq \pi\right\}$, where $K_{s}^{1}(\varphi)$ and $K_{s}^{2}(\varphi)$ are the lower and the upper bound of the mask, respectively. Furthermore, we require that each of the $S$ array patterns can be transformed into any of the others by keeping constant the excitation amplitude of each array element (phase-only control). Finally, we impose that the electric field radiated by the array in correspondence of $\mathbf{i}_{s}, \overline{\mathbf{E}}\left(\mathbf{i}_{s} ; \overline{\mathbf{r}}\right)$, be zero in $M$ suitable points $\overline{\mathbf{r}}_{m}$ located in the near-field region, with $M<\frac{N}{3}$. If such points are close to each other, then the field reduction is achieved in a neighborhood of them.

This problem can be formalized as follows: find $\mathbf{i}_{s}=$ $\left[i_{1 s}, \ldots, i_{N s}\right]^{T}, s=1, \ldots, S$, in such a way as to satisfy the following constraints:

$$
\begin{aligned}
& F\left(\mathbf{i}_{s} ; \varphi\right) \in K_{s}, s=1, \ldots, S \\
& \left|i_{n 1}\right|=\cdots=\left|i_{n S}\right|, n=1, \ldots, N \\
& \overline{\mathbf{E}}\left(\mathbf{i}_{s} ; \overline{\mathbf{r}}_{m}\right)=0, s=1, \ldots, S, m=1, \ldots, M .
\end{aligned}
$$

We want to specify that the problem and the synthesis procedure are here formulated and described in the $x y$-plane for simplicity. However, the extension to the $(\vartheta, \varphi)$-space is straightforward. The array pattern $F\left(\mathbf{i}_{s} ; \varphi\right)$ is given by:

$$
F\left(\mathbf{i}_{s} ; \varphi\right)=\sum_{n=1}^{N} i_{n s} F_{n}(\varphi),
$$

where $F_{n}(\varphi)$ is the array pattern corresponding to the excitation vector $\mathbf{e}_{n}=[0, \ldots, 1, \ldots, 0]^{T}$ having the unity in the $n$-th position (active element pattern). The electric field $\overline{\mathbf{E}}\left(\mathbf{i}_{s} ; \overline{\mathbf{r}}\right)$ is given by:

$$
\overline{\mathbf{E}}\left(\mathbf{i}_{s} ; \overline{\mathbf{r}}\right)=\sum_{n=1}^{N} i_{n s} \overline{\mathbf{E}}_{n}(\overline{\mathbf{r}})
$$

where $\overline{\mathbf{E}}_{n}(\overline{\mathbf{r}})$ is the electric field radiated by the array in correspondence of the excitation vector $\mathbf{e}_{n}$. The constraint (1) imposes the mask requirements, with the aim of generating $S$ patterns, each having a desired shape. Constraint (2) imposes the phase-only control. Condition (3) imposes that the electric field vanishes at $M$ prescribed points $\overline{\mathbf{r}}_{m}$ located in the near-field region. If such points are close to each other, the electric field amplitude will exhibit a strong reduction in a neighborhood of them, as will be shown by the numerical example. The reason for the requirement $M<\frac{N}{3}$ will be explained below.

The method of solution that we are presenting is an evolution of that proposed in [27], and is based on the alternating projections method. We first formulate the problem as an intersection finding problem. To do so, we introduce the set $\mathscr{H}=\left\{\hat{\mathbf{h}}=\left(g_{1}(\varphi), \ldots, g_{S}(\varphi), \mathbf{h}_{1}, \ldots, \mathbf{h}_{S}\right)\right\}$ where each $g_{s}(\varphi)$ is an arbitrary complex scalar function defined in the interval $[-\pi, \pi]$ with square integrable modulus, and $\mathbf{h}_{s}=\left[h_{1 s}, \ldots, h_{N s}\right]^{T}$ is a column vector with $N$ arbitrary complex components. We define the scalar product between two elements $\hat{\mathbf{h}}, \hat{\mathbf{h}}^{\prime} \in \mathscr{H}$ as:

$$
<\hat{\mathbf{h}}, \hat{\mathbf{h}}^{\prime}>_{\mathscr{H}}=\sum_{s=1}^{S}<g_{s}(\varphi), g_{s}^{\prime}(\varphi)>+\sum_{s=1}^{S} \mathbf{h}_{\mathbf{s}}^{\prime \mathbf{H}} \mathbf{h}_{\mathbf{s}},
$$

where the superscript indicates the components of the column vector $\hat{\mathbf{h}}^{\prime},<g_{s}(\varphi), g_{s}^{\prime}(\varphi)>=\int_{-\pi}^{\pi} g_{s}(\varphi) g_{s}^{\prime *}(\varphi) \mathrm{d} \varphi$, the asterisk denotes the complex conjugate and the superscript $H$ denotes transpose conjugate. The scalar product in Eq. (6) yields the norm $\|\hat{\mathbf{h}}\|_{\mathscr{H}}=\sqrt{<\hat{\mathbf{h}}, \hat{\mathbf{h}}\rangle_{\mathscr{H}}}$ and the distance $\rho\left(\hat{\mathbf{h}}, \hat{\mathbf{h}}^{\prime}\right)=\left\|\hat{\mathbf{h}}-\hat{\mathbf{h}}^{\prime}\right\|_{\mathscr{H}}$, which becomes

$$
\begin{aligned}
\rho^{2}\left(\hat{\mathbf{h}}, \hat{\mathbf{h}}^{\prime}\right) & =\sum_{s=1}^{S} \int_{-\pi}^{\pi} \mid\left(g_{s}(\varphi)-\left.g_{s}^{\prime}(\varphi)\right|^{2} \mathrm{~d} \varphi+\right. \\
& +\sum_{s=1}^{S} \sum_{n=1}^{N}\left|h_{n s}-h_{n s}^{\prime}\right|^{2} .
\end{aligned}
$$

In $\mathscr{H}$ we now introduce the sets:

$$
\begin{gathered}
K=\left\{\hat{\mathbf{k}}=\left(f_{1}(\varphi), \ldots, f_{S}(\varphi), \mathbf{k}_{1}, \ldots, \mathbf{k}_{s}\right):\right. \\
f_{S}(\varphi) \in K_{s}, \text { and }\left|k_{n 1}\right|=\cdots=\left|k_{n S}\right|, \\
s=1, \ldots, S, n=1, \ldots, N\} \\
W=\left\{\hat{\mathbf{w}}=\left(F\left(\mathbf{w}_{1} ; \varphi\right), \ldots, F\left(\mathbf{w}_{S} ; \varphi\right), \mathbf{w}_{1}, \ldots, \mathbf{w}_{S}\right)\right\}
\end{gathered}
$$

and

$$
\begin{aligned}
Z=\{\hat{\mathbf{z}}= & \left(F\left(\mathbf{z}_{1} ; \varphi\right), \ldots, F\left(\mathbf{z}_{S} ; \varphi\right), \mathbf{z}_{1}, \ldots, \mathbf{z}_{S}\right): \\
& \left.\overline{\mathbf{E}}\left(\mathbf{z}_{S} ; \overline{\mathbf{r}}_{\mathbf{m}}\right)=0, s=1, \ldots, S, m=1, \ldots, M\right\} .(10)
\end{aligned}
$$

Note that the elements of $K$ consist of $S$ arbitrary scalar complex functions and of $S$ arbitrary complex vectors satisfying (1) and (2), respectively, but the functions $f_{s}(\varphi)$ are not array patterns. Instead, the elements of $W$ consist of $S$ array patterns and of $S$ excitation vectors that produce such patterns, but such elements do not satisfy constraints (1) and (2). The set $Z$ consists of those elements of $W$ whose electric field vectors satisfy condition (3). It becomes now evident that each element belonging to the intersection $K \cap Z$ is a solution to our problem. Since such an intersection may be empty, we consider as a solution an element of $K$ minimizing the distance from $Z$. Such solution can be sought with the alternating projections method as described below.

Starting from a suitable point $\hat{\mathbf{k}}_{0} \in K$, we follow the iteration scheme:

$$
\hat{\mathbf{k}}_{n+1}=T_{K} T_{Z}\left[\hat{\mathbf{k}}_{n}\right], \quad n=0,1,2, \ldots
$$

where $T_{K}$ and $T_{Z}$ are the projection operators onto the sets $K$ and $Z$, respectively. By definition of distance, and due to $T_{K}$ and $T_{Z}$ being two projectors, it results: $\rho_{n} \geq \rho_{n+1}$ [12], where $\rho_{n}$ is the distance from $\hat{\mathbf{k}}_{n}$ to the set $Z$. In other words, the non-negative sequence $\left\{\rho_{n}\right\}$ is non-increasing, and therefore is convergent. The 
iteration (11) generates a sequence of points $\hat{\mathbf{k}}_{n}$ of $K$, which are closer and closer to $Z$. We stop the procedure at a point $\hat{\mathbf{k}}_{n}^{o}=\left(f_{1}^{o}(\varphi), \ldots, f_{S}^{o}(\varphi), \mathbf{k}_{1}^{o}, \ldots, \mathbf{k}_{S}^{o}\right)$ such that:

$$
\rho_{n}<\varepsilon \quad \text { or } \quad \frac{\left(\rho_{n-1}-\rho_{n}\right)}{\rho_{n}}<\delta
$$

with $\varepsilon$ and $\delta$ two suitable thresholds. As the optimal excitation vectors we consider the $S$ vector components $\mathbf{k}_{s}^{o}$ of $\hat{\mathbf{k}}_{n}^{o}$ which (being $\hat{\mathbf{k}}_{n}^{o} \in K$ ) satisfy (2) rigorously, so that phase-only control is guaranteed. The optimal radiation patterns and the radiated fields are calculated replacing $\mathbf{i}_{s}$ with $\mathbf{k}_{s}^{o}$ in (4) and (5), respectively. With this choice, constraints (1) and (3) are satisfied only approximately. However, the accuracy is very good, as will be shown by the numerical example.

\section{The Projector Operators}

In this section, formulas to implement the above projectors are derived. We recall that the projector $T_{C}$ onto the closed set $C \subset \mathscr{H}$ is the operator that associates with each point $\hat{\mathbf{h}} \in \mathscr{H}$ the point $\hat{\mathbf{c}} \in C$ closest to $\hat{\mathbf{h}}$, that is

$$
T_{C}: \hat{\mathbf{h}} \in \mathscr{H} \mapsto T_{C}[\hat{\mathbf{h}}]=\hat{\mathbf{c}} \in C: \rho(\hat{\mathbf{c}}, \hat{\mathbf{h}})=\min _{\hat{\mathbf{y}} \in C}\{\rho(\hat{\mathbf{y}}, \hat{\mathbf{h}})\} .
$$

\subsection{The Projector $T_{K}$}

It is easy to show [12] that $\hat{\mathbf{k}}=\left(f_{1}(\varphi), \ldots, f_{S}(\varphi), \mathbf{k}_{1}, \ldots, \mathbf{k}_{S}\right)$ is the projection of $\hat{\mathbf{h}}=\left(g_{1}(\varphi), \ldots, g_{S}(\varphi), \mathbf{h}_{1}, \ldots, \mathbf{h}_{S}\right)$ onto the set $K$ if for each $s=1, \ldots, S$, it results:

$$
f_{s}(\varphi)=\left\{\begin{array}{llr}
g_{s}(\varphi) & \text { if } & K_{s}^{1}(\varphi) \leq\left|g_{s}(\varphi)\right| \leq K_{s}^{2}(\varphi) \\
K_{s}^{1}(\varphi) \frac{g_{s}(\varphi)}{\left|g_{s}(\varphi)\right|} & \text { if } & \left|g_{s}(\varphi)\right|<K_{s}^{1}(\varphi) \\
K_{s}^{2}(\varphi) \frac{g_{s}(\varphi)}{\left|g_{s}(\varphi)\right|} & \text { if } & \left|g_{s}(\varphi)\right|>K_{s}^{2}(\varphi)
\end{array}\right.
$$

and $\mathbf{k}_{s}=\left[k_{1} \mathrm{e}^{j \varphi_{1 s}}, \ldots, k_{N} \mathrm{e}^{j \varphi_{N s}}\right]^{T}$, where for $n=1, \ldots, N$, $k_{n}=\frac{1}{S} \sum_{s=1}^{S}\left|h_{n s}\right|$ is the amplitude of the $n$-th array element and $\varphi_{n s}=\arg \left\{h_{n s}\right\}$ is the phase of the $n$-th element necessary to radiate the $s$-th pattern.

\subsection{The Projector $T_{W}$}

Let us note [27] that $T_{Z}[\hat{\mathbf{h}}]=T_{Z}\left[T_{W}[\hat{\mathbf{h}}]\right]$, so before expressing $T_{Z}$ we express $T_{W}$. The projection of $\hat{\mathbf{h}}=\left(g_{1}(\varphi), \ldots, g_{S}(\varphi)\right.$, $\left.\mathbf{h}_{1}, \ldots, \mathbf{h}_{S}\right)$ onto the set $W$ is the point $T_{W}[\hat{\mathbf{h}}]=\hat{\mathbf{w}}=$ $\left(y_{1}(\varphi), \ldots y_{S}(\varphi), \mathbf{w}_{1}, \ldots, \mathbf{w}_{S}\right)$, which minimizes the distance $\rho_{W}\left(\mathbf{w}_{1}, \ldots, \mathbf{w}_{S}\right)=\rho(\hat{\mathbf{w}}, \hat{\mathbf{h}})$. From the definition of scalar product and distance in $\mathscr{H}$ (see Section 2 and Eqs. (6) and (7)), it results:

$$
\begin{aligned}
\rho_{W}^{2}\left(\mathbf{w}_{1}, \ldots, \mathbf{w}_{S}\right) & =\sum_{s=1}^{S}<y_{s}(\varphi)-g_{s}(\varphi), y_{s}(\varphi)-g_{s}(\varphi)>+ \\
& +\sum_{s=1}^{S}\left(\mathbf{w}_{s}-\mathbf{h}_{s}\right)^{H}\left(\mathbf{w}_{s}-\mathbf{h}_{s}\right) .
\end{aligned}
$$

By definition (9) of $W$, after some manipulations we find:

$$
\begin{gathered}
\rho_{W}^{2}\left(\mathbf{w}_{1}, \ldots, \mathbf{w}_{S}\right)=\sum_{s=1}^{S}\left\{\mathbf{w}_{s}^{H} \mathbf{F} \mathbf{w}_{s}-\mathbf{w}_{s}^{H} \mathbf{g}_{s}-\mathbf{g}_{s}^{H} \mathbf{w}_{s}+\mathbf{g}_{s}^{H} \mathbf{g}_{s}\right\}+ \\
+\sum_{s=1}^{S}\left\{\mathbf{w}_{s}^{H} \mathbf{w}_{s}-\mathbf{w}_{s}^{H} \mathbf{h}_{s}\right\}-\sum_{s=1}^{S}\left\{\mathbf{h}_{s}^{H} \mathbf{w}_{s}-\mathbf{h}_{s}^{H} \mathbf{h}_{s}\right\}
\end{gathered}
$$

where $\mathbf{F}=\left[F_{m n}\right]$, with $F_{m n}=\int_{-\pi}^{\pi} F_{n}(\varphi) F_{m}^{*}(\varphi) \mathrm{d} \varphi$ and $\mathbf{g}_{s}$ is the $s$-th column of the $[N \times S]$ matrix $\mathbf{G}=\left[g_{m s}\right]$ with $g_{m s}=\int_{-\pi}^{\pi} g_{s}(\varphi) F_{m}^{*}(\varphi) \mathrm{d} \varphi$. Imposing the condition $\frac{\partial \rho_{W}^{2}}{\partial w_{p s}^{*}}=0$, $p=1, \ldots, N, s=1, \ldots, S$, yields the $S$ matrix equations:

$$
\mathbf{J w}_{s}=\mathbf{m}_{s}
$$

or equivalently the matrix equation:

$$
\mathbf{J W}=\mathbf{M},
$$

where $\mathbf{J}=\mathbf{F}+\mathbf{I}_{\mathrm{N}}, \mathbf{M}=\mathbf{G}+\mathbf{H}$, being $\mathbf{I}_{N}$ the identity matrix of rank $N, \mathbf{W}$ and $\mathbf{H}$ the $[N \times S]$ matrices whose $s$-th columns are, respectively, $\mathbf{w}_{s}$ and $\mathbf{h}_{s}$. The solution to (18) is

$$
\mathbf{W}=\mathbf{J}^{\dagger} \mathbf{M}
$$

where $\mathbf{J}^{\dagger}$ is the pseudo-inverse matrix of $\mathbf{J}$ (which coincides with the inverse matrix $\mathbf{J}^{-1}$ if $\mathbf{J}$ is non-singular [28]). Once we have the vectors $\mathbf{w}_{s}$, with the definition (9) of $W$ we can calculate the functions $y_{s}(\varphi)=\sum_{n=1}^{N} w_{n s} F_{n}(\varphi)$, hence the projection $\hat{\mathbf{w}}=\left(y_{1}(\varphi), \ldots, y_{S}(\varphi), \mathbf{w}_{1}, \ldots, \mathbf{w}_{S}\right)=T_{W}[\hat{\mathbf{h}}]$.

\subsection{The Projector $T_{Z}$}

Now, in order to implement the projector $T_{Z}$, we have to minimize the quantity $\rho_{Z}\left(\mathbf{z}_{1}, \ldots, \mathbf{z}_{S}\right)=\rho(\hat{\mathbf{z}}, \hat{\mathbf{h}})$ or, equivalently, $\rho\left(\hat{\mathbf{z}}, T_{W}[\hat{\mathbf{h}}]\right)=\rho\left(\hat{\mathbf{z}}, \hat{\mathbf{w}}_{0}\right)$, where $\hat{\mathbf{w}}_{0}=T_{W}[\hat{\mathbf{h}}]$. By definition (10) of $Z$, after some manipulations we have:

$$
\rho_{Z}^{2}\left(\hat{\mathbf{z}}, \hat{\mathbf{w}}_{0}\right)=\sum_{s=1}^{S}\left(\mathbf{z}_{s}-\mathbf{w}_{0_{s}}\right)^{H} \mathbf{J}\left(\mathbf{z}_{s}-\mathbf{w}_{0_{s}}\right),
$$

where $\mathbf{w}_{0 s}$ is the $s$-th excitation vector of $\hat{\mathbf{w}}_{0}$. Note that, by (17),

$$
\mathbf{w}_{0_{s}}=\mathbf{J}^{\dagger} \mathbf{m}_{s},
$$

Since $\mathbf{F}$ and $\mathbf{I}_{N}$ are Hermitian, also $\mathbf{J}=\mathbf{F}+\mathbf{I}_{N}$ is Hermitian and hence all eigenvalues of $\mathbf{J}$ are real and $\mathbf{J}$ is unitarily diagonalizable [28]. Therefore $\mathbf{J}=\mathbf{U}^{H} \Lambda \mathbf{U}$, where $\Lambda$ is the diagonal matrix of the eigenvalues of $\mathbf{J}$, the columns of $\mathbf{U}^{H}$ are the corresponding eigenvectors, and $\mathbf{U}^{H}$ is unitary. Replacing $\mathbf{J}$ with $\mathbf{U}^{H} \Lambda \mathbf{U}$ yields:

$$
\rho_{Z}^{2}\left(\hat{\mathbf{z}}, \hat{\mathbf{w}}_{0}\right)=\sum_{s=1}^{S}\left(\mathbf{z}_{s}-\mathbf{w}_{0_{s}}\right)^{H} \mathbf{U}^{H} \Lambda \mathbf{U}\left(\mathbf{z}_{s}-\mathbf{w}_{0_{s}}\right),
$$

which can be written as:

$$
\rho^{2}\left(\hat{\mathbf{z}}, \hat{\mathbf{w}}_{0}\right)=\sum_{s=1}^{S}\left(\mathbf{v}_{s}-\mathbf{v}_{0_{s}}\right)^{H}\left(\mathbf{v}_{s}-\mathbf{v}_{0_{s}}\right)=\sum_{s=1}^{S}\left\|\mathbf{v}_{s}-\mathbf{v}_{0_{s}}\right\|_{E}^{2},
$$


where we set

$$
\mathbf{v}_{s}=\Lambda^{\frac{1}{2}} \mathbf{U} \mathbf{z}_{s} \quad \text { and } \quad \mathbf{v}_{0_{s}}=\Lambda^{\frac{1}{2}} \mathbf{U} \mathbf{w}_{0_{s}} .
$$

So, projecting $\hat{\mathbf{h}}$ onto $Z$ reduces to minimize the quantity (23) subject to the constraint (3). Now, constraint (3) can be formulated due the matrix equations

$$
\mathbf{E} \mathbf{z}_{s}=\mathbf{E} \mathbf{U}^{-1} \Lambda^{-\frac{1}{2}} \mathbf{v}_{s}=\mathbf{S} \mathbf{v}_{s}=0
$$

where $\mathbf{E}$ denotes the $3 M \times N$ matrix defined as follows:

$$
\mathbf{E}=\left[\begin{array}{c}
\mathbf{E}_{x} \\
\mathbf{E}_{y} \\
\mathbf{E}_{z}
\end{array}\right]
$$

with $\mathbf{E}_{x}, \mathbf{E}_{y}, \mathbf{E}_{z}$ being the three $M \times N$ matrices whose entries are $E_{x_{m n}}=E_{x_{n}}\left(\overline{\mathbf{r}}_{m}\right), E_{y_{m n}}=E_{y_{n}}\left(\overline{\mathbf{r}}_{m}\right), E_{z_{m n}}=E_{z_{n}}\left(\overline{\mathbf{r}}_{m}\right)$, respectively, with $E_{\xi_{n}}\left(\overline{\mathbf{r}}_{m}\right)$ denoting the $\xi$-component $(\xi=$ $x, y, z)$ of the electric field produced at the point $\overline{\mathbf{r}}_{m}$ by the array with excitation vector $\mathbf{e}_{n}$. The matrix $\mathbf{S}=\mathbf{E} \mathbf{U}^{-1} \Lambda^{-\frac{1}{2}}$ has $3 M$ rows and $N$ columns, thus Eq. (25), expressing the near-field constraints, has a solution if $M<\frac{N}{3}$. The vectors $\mathbf{v}_{S}$ minimizing (23) and satisfying (25) are:

$$
\mathbf{v}_{s}=\left(\mathbf{I}_{N}-\mathbf{S}^{\dagger} \mathbf{S}\right) \mathbf{v}_{0_{s}} .
$$

Therefore, recalling Eq. (24) yields:

$$
\mathbf{z}_{s}=\mathbf{P} \mathbf{w}_{0_{s}}, \quad s=1, \ldots, S,
$$

where $\mathbf{P}=\mathbf{U}^{-1} \Lambda^{-\frac{1}{2}}\left(\mathbf{I}_{N}-\mathbf{S}^{\dagger} \mathbf{S}\right) \Lambda^{\frac{1}{2}} \mathbf{U}$ and $\mathbf{w}_{0_{s}}$ is given by Eq. (21), which solves Eq. (17). With the vectors $\mathbf{z}_{S}$ and the definition (10) of $Z$, we can calculate the functions $F\left(\mathbf{z}_{\mathbf{s}}, \varphi\right)=\sum_{n=1}^{N} z_{n s} F_{n}(\varphi)$, hence the projection $\hat{\mathbf{z}}=$ $\left(F\left(\mathbf{z}_{1}, \varphi\right), \ldots, F\left(\mathbf{z}_{S}, \varphi\right), \mathbf{z}_{1}, \ldots, \mathbf{z}_{S}\right)=T_{Z}[\hat{\mathbf{h}}]$.

\section{Numerical Example}

We considered the array shown in Fig. 1, consisting of $N=429$ elementary vertical dipoles with length $l=\frac{\lambda}{50}$.

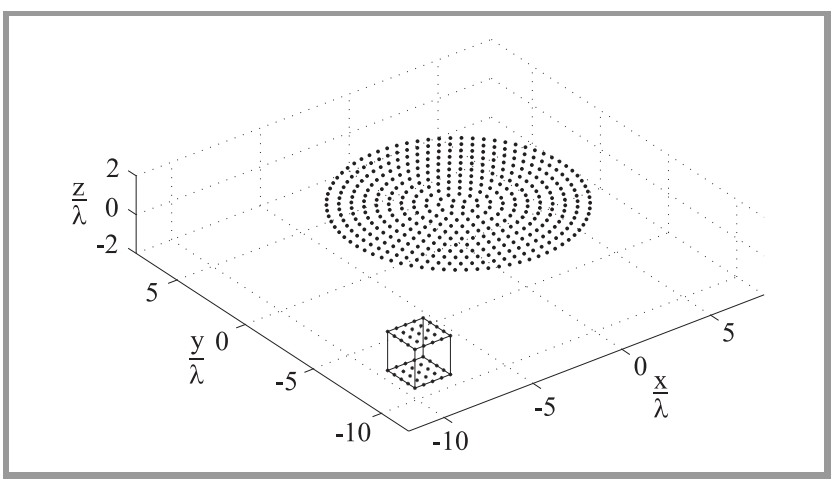

Fig. 1. Array geometry and near-field region for the proposed example. The null points lie on the top and bottom faces of the cube.
The elements are equally spaced on 11 circular rings (as it is described in Table 1), with each ring having the center at the origin of the Cartesian system $O(x, y, z)$ and lying on the $x y$-plane. In the near-field region, on the planes $z= \pm \lambda$, two meshes were defined, consisting of 25 equally spaced points (see Fig. 1), with $-10.5 \lambda \leq x, y \leq-8.5 \lambda$, resulting in $M=50$ points $\overline{\mathbf{r}}_{m}$ with a minimum distance between adjacent points of $\frac{\lambda}{2}$. Note also that $M=50<143=\frac{N}{3}$. The array was required to synthesize by phase-only control the $S=4$ patterns of Fig. 2 .

Table 1

Array geometry details: normalized radius $\frac{R_{i}}{\lambda}$ and number of elements $N_{i}$ of each ring

\begin{tabular}{|c|c|c|c|c|c|c|c|c|}
\hline$i$ & $\frac{R_{i}}{\lambda}$ & $N_{i}$ & $i$ & $\frac{R_{i}}{\lambda}$ & $N_{i}$ & $i$ & $\frac{R_{i}}{\lambda}$ & $N_{i}$ \\
\hline \hline 1 & 0.35 & 4 & 5 & 2.55 & 32 & 9 & 4.77 & 60 \\
\hline 2 & 0.88 & 11 & 6 & 3.11 & 39 & 10 & 5.33 & 67 \\
\hline 3 & 1.44 & 18 & 7 & 3.66 & 46 & 11 & 5.8 & 74 \\
\hline 4 & 1.99 & 25 & 8 & 4.22 & 53 & & & \\
\hline
\end{tabular}

First, the synthesis was performed without imposing the near-field nulls, that is, in the absence of condition (3). This was realized by replacing the projector $T_{Z}$ in Eq. (11) with $T_{W}$. In the following it will be referred to as the "reduced" problem. Then constraint (3) was taken into account and the "complete" problem was solved.

Both the reduced and the complete problem gave very good results in a satisfactory computer time. In fact, the former required only $1.78 \mathrm{~s}$ (corresponding to 104 iterations) and the latter required $30.19 \mathrm{~s}$ (1761 iterations). As it was expected, constraint (2) was satisfied exactly. Condition (1) was very well approximated. In fact, the synthesized patterns exceeded the mask limits at most of $0.18 \mathrm{~dB}$ (reduced problem) and $0.03 \mathrm{~dB}$ (complete problem), and in particular, the maximum side lobe levels in the worst case were $-34.82 \mathrm{~dB}$ (reduced problem) and $-34.97 \mathrm{~dB}$ (complete problem). Figure 2 shows the radiation patterns obtained solving the complete problem. Figure 3 shows a contour plot of the near-field amplitude in both the reduced and the complete problem. Constraint (3) was approximated quite satisfactorily, as the maximum near-field amplitude on the constraint points in the complete problem exhibited a $50.03 \mathrm{~dB}$ reduction with respect to that of the reduced problem. Finally, the field amplitudes were evaluated on a mesh of $\frac{\lambda}{8}$ spaced points in the cube of Fig. 1 for both the reduced and the com-

Table 2

Near-field amplitude comparison

\begin{tabular}{|l|c|}
\hline Reduction of the maximum amplitude & $48.25 \mathrm{~dB}$ \\
\hline Reduction of the mean amplitude & $43.06 \mathrm{~dB}$ \\
\hline Maximum reduction & $58.62 \mathrm{~dB}$ \\
\hline Minimum reduction & $15.86 \mathrm{~dB}$ \\
\hline Mean reduction & $39.42 \mathrm{~dB}$ \\
\hline
\end{tabular}



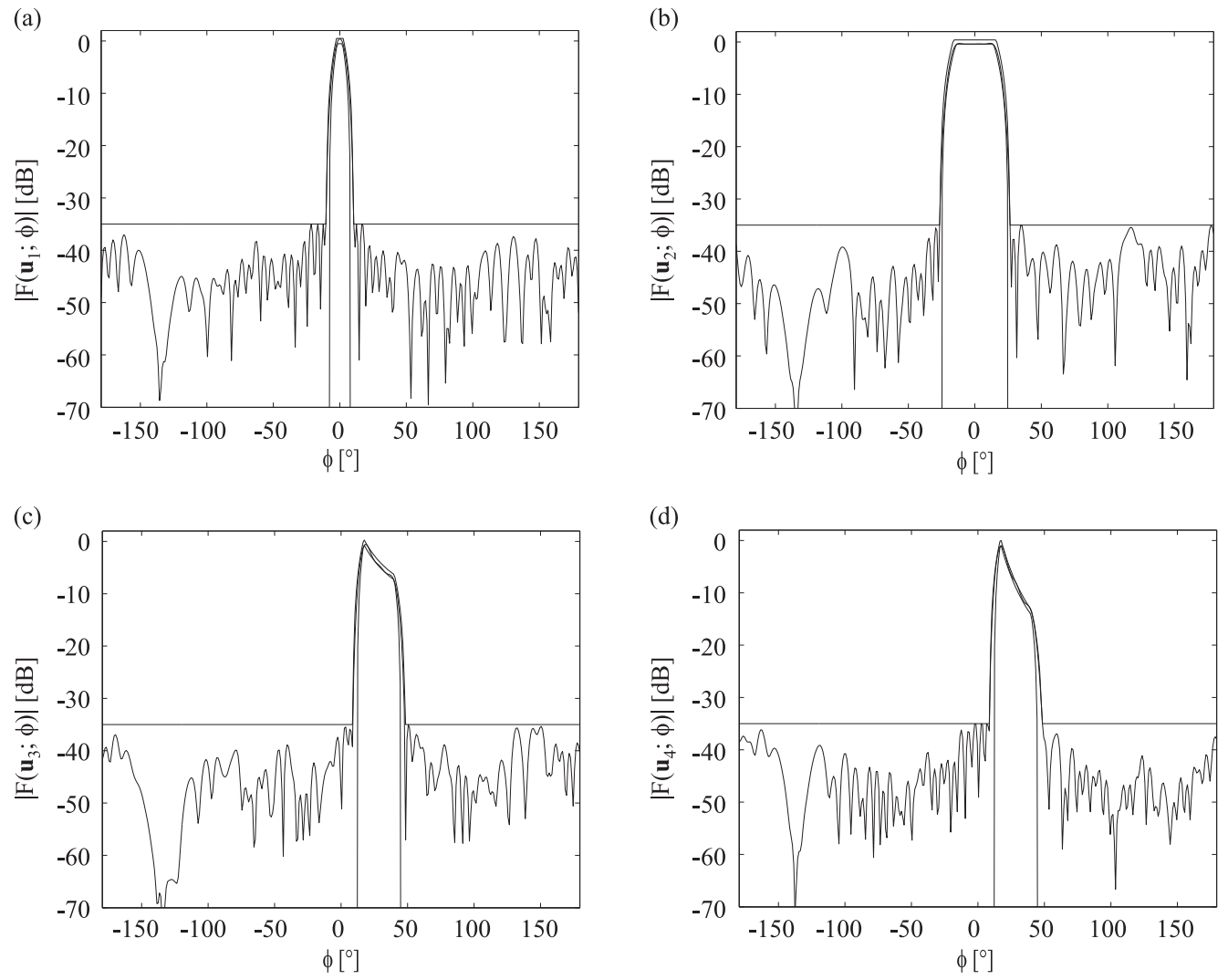

Fig. 2. The assigned masks and the synthesized patterns for the complete problem: (a) a pencil beam, (b) a flat top beam), (c) a cosecant, (d) a squared cosecant.
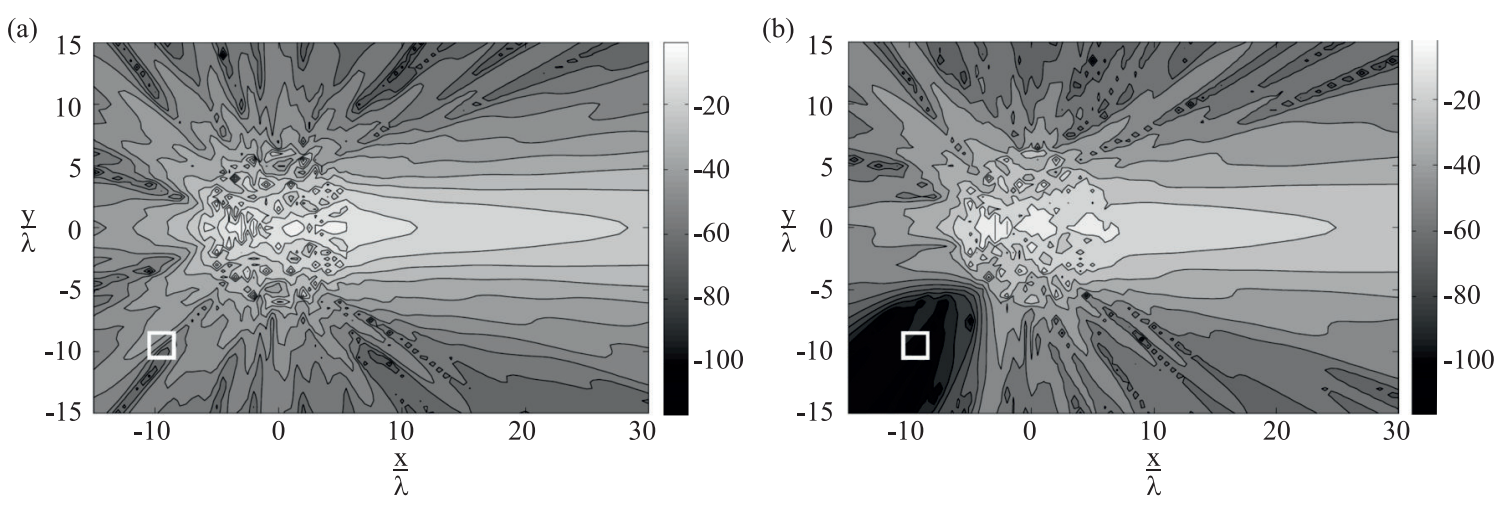

Fig. 3. Contour plot of the near-field amplitude (in $\mathrm{dB}$ ) on a portion of the $x y$-plane, obtained in the reduced (a) and complete (b) problem.

plete problem. The results are summarized in Table 2 and show that imposing near-field nulls on a number of suitably located points allows to obtain a very strong reduction of the electric field amplitude in the whole region of interest.

\section{Conclusions}

The algorithm presented in this paper allows us to synthesize reconfigurable antenna arrays with phase-only control, simultaneously reducing the near-field amplitude in a region close to the antenna. The near-field reduction is obtained by imposing that the field vanishes at a prescribed number of suitably located points. In such a way, strong reductions can be obtained without increasing the dimensions of the problem and so keeping low the required computational time. This represents one of the main advantages of the presented algorithm with respect to those presented in [20], [21], which allow to control the near-field reduction, but with a higher computational time. 


\section{Acknowledgements}

We thank the Guest Editors Lara Pajewski, Hovik Baghdasaryan, and Marian Marciniak for inviting us to participate in the JTIT Special Issue "Recent Progress in Electromagnetic Theory and its Applications" organized by the COST Action TU1208 "Civil engineering applications of Ground Penetrating Radar".

\section{References}

[1] G. Buttazzoni and R. Vescovo, "Deterministic and stochastic approach to the synthesis of conformal arrays for SAR applications", in Proc. 2013 Int. Conf. on Electromag. in Adv. Appl. ICEAA 2013, Torino, Italy, 2013, pp. 520-523.

[2] R. K. Singh, A. Basu, and S. K. Koul, "Efficient null broadening and steering using slot antenna array for radar applications", in Proc. 2016 Asia-Pacific Microwave Conf. APMC 2016, New Delhi, India, 2016, pp. 1-4.

[3] G. Buttazzoni, M. Comisso, A. Cuttin, M. Fragiacomo, R. Vescovo, and R. Vincenti Gatti, "Reconfigurable phased antenna array for extending cubesat operations to Ka-band: Design and feasibility", Acta Astronautica, vol. 137, pp. 114-121, 2017.

[4] J. Huang, F. Qiu, W. Lin, Z. Tang, D. Lei, M. Yao, Q. X. Chu, and Y. J. Guo, "A new compact and high gain circularly-polarized slot antenna array for Ku-band mobile satellite TV reception", IEEE Access, vol. 5, pp. 6707-6714, 2017.

[5] Q. U. A. Nadeem, A. Kammoun, M. Debbah, and M. S. Alouini, "Performance analysis of compact FD-MIMO antenna arrays in a correlated environment", IEEE Access, vol. 5, pp. 4163-4178, 2017.

[6] O. M. Haraz, A. Elboushi, S. A. Alshebeili, and A. R. Sebak, "Dense dielectric patch array antenna with improved radiation characteristics using EBG Ground structure and dielectric superstrate for future 5G cellular networks", IEEE Access, vol. 2, pp. 909-913, 2014.

[7] L. I. Vaskelainen, "Phase synthesis of conformal array antennas", IEEE Trans. Antennas Propag., vol. 48, no. 6, pp. 987-991, 2000.

[8] M. Durr, A. Trastoy, and F. Ares, "Multiple-pattern linear antenna arrays with single prefixed amplitude distributions: modified Woodward-Lawson synthesis", Electron. Lett., vol. 36, no. 16, pp. 1345-1346, 2000.

[9] O. M. Bucci, G. Mazzarella, and G. Panariello, "Reconfigurable arrays by phase-only control", IEEE Trans. Antennas Propag., vol. 39, no. 7, pp. 919-925, 1991.

[10] O. M. Bucci and G. D'Elia, "Power synthesis of reconfigurable conformal arrays with phase-only control", IEE Proc. Microw. Antennas Propag., vol. 145, no. 1, pp. 131-136, 1998.

[11] G. K. Mahanti and A. Chakraborty, "Phase-only and amplitudephase synthesis of dual-pattern linear antenna arrays using floatingpoint genetic algorithms", Progress in Electromag. Res., PIER 68, pp. 247-259, 2007.

[12] R. Vescovo, "Reconfigurability and beam-scanning with phase-only control for antenna arrays", IEEE Trans. Antennas Propag., vol. 56, no. 6, pp. 1555-1565, 2008.

[13] G. Buttazzoni and R. Vescovo, "Synthesis of co-polar and crosspolar patterns with dynamic range ratio reduction for phase-only reconfigurable arrays", in Proc. 6th Eur. Conf. on Antenn. and Propag. EUCAP 2012, Prague, Czech Republic, 2012, pp. 2623-2627.

[14] F. Obelleiro, L. Landesa, J. L. Rodriguez, A. G. Pino, and M. R. Pino, "Directivity optimisation of an array antenna with obstacles within its near field region", Electron. Lett., vol. 33, no. 25, pp. 2087-2088, 1997.

[15] L. Landesa and F. Obelleiro, "Maximising directivity of array antennas mounted over complex environments with near-field null constraints", Electron. Lett., vol. 37, no. 2, pp. 74-76, 2001.

[16] L. Landesa, F. Obelleiro, J. L. Rodriguez, J. A. Rodriguez, F. Ares, and A. G. Pino, "Pattern synthesis of array antennas with additional isolation of near field arbitrary objects", Electron. Lett., vol. 34, no. 16 , pp. 1540-1542, 1998.
[17] R. Vescovo, "Pattern synthesis with assigned field reduction in nearfield for circular arrays", in Proc. IEEE Antenn. and Propag. Soc. Int. Symp., San Antonio, TX, USA, 2002, vol. 1, pp. 540-543.

[18] M. Comisso and R. Vescovo, "Fast power pattern synthesis with near-field control for antenna arrays", in Proc. EEE Antenn. and Propag. Soc. Int. Symp., Charleston, SC, USA, 2009, pp. 1-4.

[19] M. Comisso, G. Buttazzoni, and R. Vescovo, "Reconfigurable antenna arrays with multiple requirements: A versatile 3D approach", Int. J. of Antenn. and Propag., vol. 2017, Article ID 6752108, 2017 (doi: 10.1155/2017/6752108).

[20] O. M. Bucci, A. Capozzoli, and G. D'Elia, "Power pattern synthesis of reconfigurable conformal arrays with near-field constraints", IEEE Trans. Antennas Propag., vol. 52, no. 1, pp. 132-141, 2004.

[21] G. Buttazzoni and R. Vescovo, "Reconfigurable array synthesis with constraints on near field, far field and dynamic range ratio", in Proc. Int. Conf. on Electromag. in Advanced Appl. ICEAA '09, Torino, Italy, 2009, pp. 257-260.

[22] G. Buttazzoni and R. Vescovo, "Phase-controlled beam-scanning with near-field and DRR reduction for arbitrary antenna arrays", in 2010 IEEE Antenn. and Propag. Soc. Int. Symp., Toronto, ON, Canada, 2010, pp. 1-4.

[23] G. Buttazzoni and R. Vescovo, "An efficient and versatile technique for the synthesis of 3D copolar and crosspolar patterns of phase-only reconfigurable conformal arrays with DRR and near-field control", IEEE Transactions on Antennas and Propagation, vol. 62, no. 4, pp. 1640-1651, 2014.

[24] G. Buttazzoni and R. Vescovo, "A deterministic approach to the synthesis of sparse arrays with far-field and near-field constraints", in Proc. 2015 IEEE-APS Topical Conf. on Antenn. and Propag. in Wireless Commun. APWC 2015, orino, Italy, 2015, pp. 161-164.

[25] H. Steyskal, "Synthesis of antenna patterns with imposed near-field nulls", Electron. Lett., vol. 30, no. 24, pp. 2000-2001, 1994.

[26] O. M. Bucci, F. D’Agostino, C. Gennarelli, G. Riccio, and C. Savarese, "Array pattern synthesis with null field constraints in the near-field region", in IEEE Antenn. and Propag. Soc. Int. Symp., vol. 3, Boston, MA, USA, 2001, pp. 716-719.

[27] R. Vescovo, "Power pattern synthesis for antenna arrays with null constraints in the near-field region", Microw. Opt. Technol. Lett., vol. 44, no. 6, pp. 542-545, 2005.

[28] R. A. Horn and C.R. Johnson, Matrix Analysis. New York: Cambridge University Press, 1992.

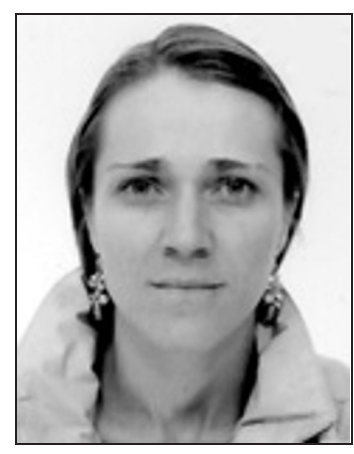

Giulia Buttazzoni received the Laurea degree (summa cum laude) in Telecommunication Engineering and the Ph.D. degree in Information Engineering from the University of Trieste (Italy), in 2008 and 2013, respectively. She is currently a researcher at the Department of Engineering and Architecture of the University of Trieste. Her research interests involve antenna array synthesis and numerical methods for electromagnetic fields.

E-mail: gbuttazzoni@units.it

Department of Engineering and Architecture

University of Trieste

Piazzale Europa 1

34127 Trieste, Italy

Roberto Vescovo - for biography, see this issue, p. 29. 